\title{
Emerging role of mitophagy in human diseases and physiology
}

\author{
Jee-Hyun $\mathrm{Um}^{1,2} \mathcal{E}$ Jeanho Yun ${ }^{1,2, *}$ \\ ${ }^{1}$ Department of Biochemistry, and ${ }^{2}$ Peripheral Neuropathy Research Center, College of Medicine, Dong-A University, Busan 49201, Korea
}

\begin{abstract}
Mitophagy is a process of selective removal of damaged or unnecessary mitochondria using autophagic machinery. Mitophagy plays an essential role in maintaining mitochondrial quality control and homeostasis. Mitochondrial dysfunctions and defective mitophagy in neurodegenerative diseases, cancer, and metabolic diseases indicate a close link between human disease and mitophagy. Furthermore, recent studies showing the involvement of mitophagy in differentiation and development, suggest that mitophagy may play a more active role in controlling cellular functions. A better understanding of mitophagy will provide insights about human disease and offer novel chance for treatment. This review mainly focuses on the recent implications for mitophagy in human diseases and normal physiology. [BMB Reports 2017; 50(6): 299-307]
\end{abstract}

\section{INTRODUCTION}

Mitochondria are essential organelles for providing energy and metabolic intermediates. In addition, mitochondria are the main sites for reactive oxygen species (ROS) generation and play central roles in cell death (1). While normal mitochondrial activities are required for cell proliferation and metabolic processes, defective mitochondrial functions have been associated with a wide variety human diseases, including cancer, diabetes mellitus and age-related disorders (2). Accumulation of mitochondrial DNA mutations and a decline in mitochondrial activity are believed to contribute to normal aging process (3). Therefore, quality control of mitochondria is a critical issue to maintain cellular function. Several mechanisms that regulate mitochondrial quality control have been identified. Mitochondria have their own resident mitochondrial chaperones and proteolytic system that help re-fold or degrade misfolded protein (4). In addition, constant mitochondrial fusion and fission process seems essential to maintain mitochondrial homeostasis via segregation of

*Corresponding author. Tel: +82-51-240-2919; Fax: +82-51-2410778; E-mail: yunj@dau.ac.kr

https://doi.org/10.5483/BMBRep.2017.50.6.056

Received 31 March 2017

Keywords: Mitophagy; human diseases; human physiology; Mitochondria dysfunctions; mitophagy activity damaged mitochondria and exchange materials (5).

Mitophagy is another critical mechanism for bulk degradation of mitochondria through a process using macroautophagy machinery. In this process, entire mitochondria are enclosed in a double-membrane vesicle, autophagosome, and delivered to lysosomes for hydrolytic degradation (6). Since John Lemasters used the term 'mitophagy' in 2005, mitophagy has been intensively studied. In particular, studies suggesting that the development of hereditary Parkinson's disease is associated with mitophagy depletion have led to an increased interest in mitophagy. In 2006, groups of Jongkyeong Chung and Ming Guo simultaneously identified that Parkinson's disease genes PINK1 and Parkin function in the same genetic pathway in Drosophila model, and in 2008, Richard Youle's group demonstrated that Parkin mediates the removal of damaged mitochondria $(7,8)$, and the PINK-Parkin pathway emerged as a key regulatory pathway of mitophagy (9). Decreased mitochondrial membrane potential $(\Delta \Psi \mathrm{m})$ induces stabilization of PINK1, followed by migration of Parkin to the mitochondria and promotes mitophagy to eliminate damaged mitochondria (10). Previous studies have well established that mitophagy is primarily responsible for the selective removal of damaged, old, or dysfunctional mitochondria. In most cases, mitophagy requires functional macroautophagy machinery, but recent observation suggests that several distinct forms of mitophagy also exist depends on types of stimuli and requirement of autophagic machinery (11).

Through recent active research on the molecular mechanism of mitophagy, mitochondrial receptors have been reported such as BNIP3, NIX/BNIP3L, FUNDC, and Atg32 in yeast, and several mitophagy regulatory proteins such as AMBRA, MUL1, AMFR, SMURF, and RHEB have been identified. Furthermore, in addition to its function of removing damaged mitochondria, mitophagy has been shown to perform a variety of physiological functions. As the molecular mechanisms and regulatory proteins on the mitophagy pathway have already been well-organized by other outstanding reviews $(6,12,13)$, this review will focus on introducing the latest research on the physiological mechanisms of mitophagy and its role in human diseases.

\section{PHYSIOLOGICAL ROLE OF MITOPHAGY}

Mitophagy in mitochondrial quality control

Mitophagy mechanism holds a central role in mitochondrial 
quality control by recognizing damages to the mitochondria and selectively removing the damaged mitochondria. In fact, various models have demonstrated that suppression or abnormalities to mitophagy result in the accumulation of damaged mitochondria (10). Mitophagy contributes to mitochondrial quality control not only by removing damaged mitochondria but also by promoting biosynthesis of new mitochondria. It has been demonstrated that there is crosstalk between the mitophagy pathway and mitochondrial biosynthetic pathway (10). Specifically, Plaikaras et al. established that mitophagy and mitochondrial biosynthesis are interfaced with each other to maintain mitochondrial homeostasis using a $C$. elegans model (14). They found that the mitochondrial biosynthetic pathway is also affected by retrograde signals upon inhibition of mitophagy. As a regulatory mechanism of the mitochondrial biosynthetic pathway by mitophagy, Shin $\mathrm{JH}$ et al. discovered that Parkin-interacting substrate (PARIS/ZNF746) inhibits transcription of PGC- $1 \alpha$, an important regulator of mitochondrial biosynthesis (15). PARIS acts as a transcriptional repressor in the nucleus and inhibits the expression of PGC-1 $\alpha$. Upon activation of mitophagy, Parkin ubiquitinates PARIS and promotes its degradation, thereby increasing transcription of PGC-1 $\alpha$ and promoting mitochondrial biosynthesis. Based on these results, there is an emerging possibility that mitochondrial quality can be improved through the facilitation of mitophagy, which leads to selective removal of damaged mitochondria and simultaneously induce the production of new mitochondria.

\section{Mitophagy in cell differentiation and development}

During differentiation and development, it is understood that the primary role of mitophagy is to remove redundant mitochondria. The most representative example is during the erythrocyte differentiation process where $\mathrm{Nix}$ acts as a mitochondrial receptor to mediate mitochondrial removal through mitophagy (16). In Nix knockout mice, mitochondria are not removed from erythrocytes, and consequently, anemia is developed due to a decrease in survival (16).

In the embryo, mitophagy has been shown to be involved in the removal of paternal mitochondria. In most eukaryotes, mitochondria are inherited maternally in offspring. Moreover, sperm mitochondria are immediately removed after fertilization. In 2011, two research groups reported that in the embryo, paternal mitochondria are removed through mitophagy in C. elegans model $(17,18)$. Upon suppression of mitophagy, paternal mitochondria lose their ability to undergo fusion with each other but are not removed. An interesting question of how sperm-derived mitochondria are selectively removed remains to be elucidated.

\section{Mitophagy in cell programming}

During the process of cell differentiation, cell reprogramming occurs in which the physiological functions of various cells such as energy metabolism and gene expression are greatly changed. Recently, it has been reported that functional changes, as well as quantitative changes in mitochondria, occur during cell differentiation. Wilson-Fritch et al. previously observed that significant increase in mitochondrial quantity during the differentiation of 3T3-L1 fibroblasts to adipocytes (19). Interestingly, in addition to quantitative changes in the mitochondria, the mitochondrial remodeling is accompanied by changes in the protein composition of the mitochondria and in the metabolic characteristics of the mitochondria. Kita $\mathrm{T}$ et al. further revealed that mitochondrial

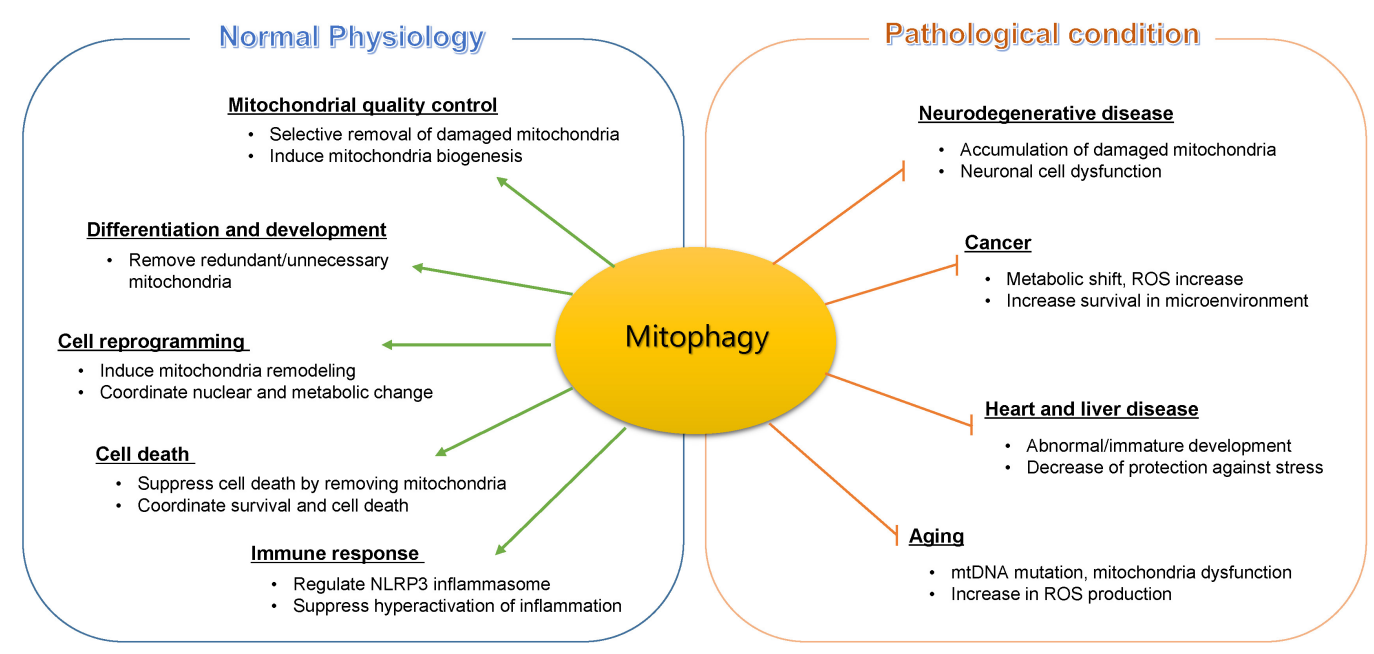

Fig. 1. The role of mitophagy in normal physiology and human disease. Mitophagy plays an important role in maintaining mitochondria homeostasis and various aspects of cellular function. These roles are critical to prevent developing human diseases and aging-related dysfunctions. 
remodeling in 3T3-L1 differentiation process is accompanied by mitochondrial fragmentation and that inhibition of fission resulted in an inhibition of fatty acid accumulation, which a representative differentiation marker (20). Because fatty acid metabolic pathway largely relies on nuclear process, these results indicate that functional change of mitochondria is closed linked to nuclear function. Interesting results that directly demonstrate the importance of mitophagy during the cell differentiation process were reported recently by the Gottlieb group (21). In C2C12 myoblast differentiation model, they found that both dramatic clearances of mitochondria and subsequent biogenesis of mitochondria occur at the early phase of differentiation. This remodeling of mitochondria is essential for a metabolic shift from glycolysis to oxidative phosphorylation and myoblast differentiation to mature myotubes. Inhibition of autophagic flux abrogates both mitochondria remodeling and myoblast differentiation, implying a pivotal role of mitophagy in $\mathrm{C} 2 \mathrm{C} 12$ myoblast differentiation.

Another representative example where mitochondrial remodeling occurs is during the differentiation process of stem cells. Nuclear reprogramming by Stemness-related transcription factors, such as Oct4, Sox2, Klf4 and c-Myc, is sufficient to reverse-differentiate fibroblasts into pluripotent stem cells (22). Interestingly, recent studies have reported that the transition of mitochondrial restructuring and energy metabolism are required for the induction of orchestrated dedifferentiation and induced pluripotent stem (iPS) cells. While parental fibroblasts have a well-developed elongated tubular-shaped mitochondrial network in the cytoplasm, in iPS, there is distribution immature mitochondria primarily in the perinuclear region that are either globular or spherical shaped and contain underdeveloped cristae (23-26). In addition, dedifferentiation into iPS cells is accompanied by metabolic remodeling by reducing mitochondrial DNA (mtDNA) copy number, reducing oxygen consumption, and increasing glycolysis. Since mitochondrial membrane potential and glycolytic genes precede the expression of pluripotent genes (23), metabolic remodeling is considered to have a causative role during the dedifferentiation process. Further studies are required to determine whether mitophagy is required in the induction process of iPS. Specifically, how mitophagy changes are linked to changes in nuclear gene expression will be an important question.

\footnotetext{
Mitophagy in cell death

Although mitochondria play an essential role in a cell's survival, concurrently, it has a role in promoting cell death. In stress conditions, the permeability of the inner mitochondrial membrane increases and releases proapoptotic molecules such as cytochrome $\mathrm{c}$ and apoptosis inducing factor (AIF) to activate the cell death pathway. Despite mitophagy's role in the suppression of cell death by removing damaged mitochondria, upon high-stress conditions and the number of damaged mitochondria increases beyond the range that can be removed by mitophagy, the cell death pathway eventually becomes
}

activated (27). Additionally, it has been identified that upon activation of apoptosis, caspase-mediated degradation of BECLIN1, AMBRA1, and others leads to suppression of mitophagy $(28,29)$. Therefore, the balance between mitophagy and the cell death pathway seems to play a critical role in determining the survival and death of cells dependent on normal and stress conditions (27). Identification of mechanisms that regulate the balance between mitophagy and cell death will provide important information in understanding the mechanisms underlying the maintenance of tissue function and disease.

\section{Mitophagy in immune response}

Recent studies have revealed the ability of mitophagy to suppress inflammation responses caused by viruses, bacteria, and others. Mitochondrial damage due to infection by foreign organisms results in the release of mitochondrial DNA and ROS and leads to the activation of inflammatory complexes such as NLRP3 (NLR family, pyrin domain containing 3). However, excessive inflammasome activation can lead to tissue damage and organ failure. Recently, it has been found that through the removal of damaged mitochondria by mitophagy, hyperactivation of NLRP3 inflammasome can be suppressed (30). Indeed, it was reported that PINK1 $1^{-1-}$ and Park2 ${ }^{-1-}$ mice were more sensitive to organ failure and death resulting from bacterial infection-mediated sepsis (31). In addition, Kim MJ et al. demonstrated that SESN2 (sestrin2) could protect against sepsis-mediated damage by inducing mitophagy (32). Further studies are required to determine the molecular mechanism of how infection induces mitochondrial damage and how mitophagy is activated by infection-mediated mitochondrial damage.

Overall, these results suggest that beyond its primary function to identify and selectively remove damaged mitochondria, mitophagy can influence various physiological processes (Fig. 1). It has already been established that mitochondria can not only receive signals from the nucleus but also send signals to the nucleus to as a mechanism to regulate gene expression. For example, unfold protein response (UPR) regulates transcription through signals sent to the nucleus as a response to increases in misfolded proteins. By specifically identifying how communication occurs between mitochondria, transcription processes of the nucleus, and other organelles, the novel functions of mitophagy will be understood.

\section{ROLE OF MITOPHAGY IN HUMAN DISEASES}

Given the pivotal role of mitophagy in mitochondrial homeostasis and quality control, it is not surprising that mitochondrial dysfunction is associated with many pathological conditions. Increasing recent studies reported defects in mitophagy process in various human diseases. 


\section{Mitophagy in neurodegenerative disease}

Mitochondrial damage and dysregulation of mitophagy have been implicated in several neurodegenerative diseases including Parkinson's disease, Alzheimer's disease, and Huntington's disease $(33,34)$. As a more direct evidence, the mutation of PINK1 and Parkin gene has been identified in hereditary Parkinson's disease patients $(35,36)$. Consistent with the critical role of PINK1 and Parkin in mitophagy, dysfunctional mitochondria are found to be accumulated in the brain of Parkinson's disease patients. The important role of PINK1 and Parkin in neuronal function was further supported by genetic studies in Drosophila model. The loss of PINK1 or Parkin function resulted in both mitochondrial dysfunction and degeneration of dopaminergic neurons $(8,37)$. Interestingly, the impairment of mitochondrial motility and mitophagy has also been recently reported in sporadic Parkinson's disease (38).

Because of the morphological nature, the maintenance of mitochondrial homeostasis is very important for the neuron. Thus, while mitophagy is thought to play a key role in the maintenance of neuronal function, studies on mitophagy within neurons are still limited. Specifically, in axons, it is unclear how damaged mitochondria are removed by mitophagy. Recently, Ashrafi G et al. reported that PINK1 and Parkin are also required to remove damaged mitochondria in neuronal axon (39). However, recent studies suggest that mitophagic clearance of damaged mitochondria in the neuron is distinct from another type of cells. Transport of the damaged axonal mitochondria by microtubule-directed motor protein and mitochondrial Rho-GTPase Miro has been shown to be important for mitophagy in axon (40), but considerable further studies are required to understand the exact molecular mechanism of neuronal mitophagy.

In addition, mitochondrial abnormalities are also commonly found in diseases of the peripheral nerves $(41,42)$. CharcotMarie-Tooth disease type 2 (CMT), the inherited axonal neuropathies, is frequently associated with mutations in proteins related to mitochondrial function such as MFN2, GDAP1 (43). Recently, Rizzo F et al. reported that motor neurons derived from iPS cells of CMT2A (MFN2) patients show defects in mitophagy (44), highlighting the importance of mitophagy activity to axonal homeostasis. Identification of the role of mitophagy in peripheral neuropathies is expected to present new therapeutic potentials.

\section{Mitophagy in cancer}

Dysregulation of mitochondrial function and accumulation of mitochondrial DNA mutations have frequently been observed in human cancers. Recent studies have suggested that functional loss of mitophagy regulators is closely linked to cancer development and progression. The close relationship between mitophagy and cancer is evident through the regulation of expression of Parkin, BNIP3, NIX, and others by representative tumor suppressors such as p53 and $\mathrm{Rb}$ and oncogenes such as NF-kB, FOXO3, and HIF-1 $\alpha$ (reviewed in (45). On the extension of this notion, recent studies have suggested that Parkin is a potential tumor suppressor. Parkin is located on chromosome $6 q 25-q 27$ that is frequently deleted in cancer $(46,47)$. It has also been observed that Parkin deletion is associated with progression of breast, colon, and liver cancer (48-50). In addition, Zhang C et al. revealed that Parkin knockout increased spontaneous liver tumor and sensitized mice to $\gamma$-irradiation (IR)-induced tumorigenesis (51). These results further support the notion that Parkin may act as a tumor suppressor. The expression of another important mitophagy regulator, PINK1, has been reported to be linked to the survival and prognosis of the adrenocortical tumor (ACT) (52, 53). It was confirmed in a mouse model that knockdown of BNIP3 and NIX promotes tumor formation and metastasis (54, 55). These results suggest that mitophagy plays a critical role to suppress cancer development. The increase of ROS could be responsible for facilitating cancer development upon mitophagy reduction. Indeed, the increase in ROS due to suppression of mitophagy has been demonstrated in the $C$. elegans model (14). Increased ROS induces DNA damage and contributes to cancer development by promoting abnormalities in gene expression.

Contrastingly, studies have also presented that an increase in mitophagy in cancer cells promotes survival and adaption in microenvironments. Hypoxia condition is commonly developed in solid tumor. The removal of mitochondria is important to lower ROS generation and maintain oxygen homeostasis. It has been reported that $\mathrm{HIF} 1 \alpha$, which plays an important role in the adaptation and survival of cancer cells to hypoxic condition, induces the expression of BNIP3 (56). Therefore, it is suggested that the increase of mitophagy is an adaptive response to hypoxia to promote the survival of cancer cells (57). As such, increases in resistance of cancer cells may be an important factor in promoting the progression of cancer cells thereby consequently increasing metastasis. Overall, the role of mitophagy in cancer development seems rather complex. While the expression of mitophagy regulatory genes is reduced in a variety of cancers, the activation of these genes leads to cancer cell proliferation and tumor growth in nude mice. Furthermore, directed studies addressing whether mitophagy plays a causal role in cancer development and progression are very limited. Identification of more precise roles of mitophagy that is dependent on the cancer type and situation is expected to present the possibility of new treatment strategies.

\section{Mitophagy in heart and liver diseases}

Various cardiac dysfunctions in mouse models deficient mitophagy regulators illustrate the importance of mitophagy in the heart. Mice deficient PINK1, BNIP3, NIX, and ATG5 developed various heart defects including cardiomyopathy, cardiac hypertrophy, and accumulation of dysfunctional mitochondria (58-60). Gong G et al. showed that Parkin- 
mediated mitophagy is essential for normal development of heart (61). Recent studies also reveal that mitophagy plays an important role in cardiac protection. Deletion of PINK1 and Parkin increased accumulation of dysfunctional mitochondria and exacerbated ischemia/reperfusion injury $(62,63)$. Ischemic preconditioning, which is the most potent intervention against ischemic injury, has been shown to induce Parkin translocation into mitochondria and require Parkin-mediated mitophagy (64). Previous studies suggest that mitophagy involves in development and maturation of heart and play an important role in cardioprotection in response to various stress.

In addition, mitochondrial dysfunction and mitophagy also associate with liver diseases including diabetes, fatty liver disease. Takamura A et al. reported that Atg7 knockout in liver led to the accumulation of abnormal, swollen mitochondria in hepatocyte (65). BNIP3 deficiency led to increased proportion of mitochondria with loss of membrane potential and elevated inflammation, steatohepatitis (66). It has also been recently suggested that mitophagy protects the liver against alcoholinduced injury (67). Thus, mitophagy is thought to protect against alcohol-induced liver injury and steatosis by selectively removing damaged mitochondria and by maintaining healthy mitochondria capable of degrading lipid through $\beta$-oxidation.

\section{Mitophagy in aging}

At both the cellular and individual level of aging, mitochondrial dysfunction and accumulation of mutations in mitochondrial DNA is commonly observed (3). The 'free radical theory' has been pointed out as the cause of aging, where mitochondrial dysfunction increases production of ROS, and through increases in mutations of the mitochondrial DNA, aging is promoted by the 'vicious cycle' in which mitochondrial dysfunction is exacerbated. Since mitophagy plays an important role in maintaining mitochondrial homeostasis, the decrease in mitophagy activity may be closely related to aging-related dysfunctions of cardiac and skeletal muscle, liver, and brain. As a direct evidence, using a mt-Keima model mouse that is capable of measuring mitophagy, a significant decrease in mitophagy activity in brain tissues of aged mice has been reported recently (68). Furthermore, it has been demonstrated that in Drosophila and C. elegans models, suppression of mitophagy decreases lifespan $(8,14)$. Studies on the mechanism of aging-dependent reduction of mitophagy and the development of mitophagy improvement methods at the individual level will present new possibilities to cope with the decline of various tissue functions associated with aging.

Recent studies proposed that targeting mitophagy would be a promising strategy for treatment of human disease. Although successful examples are still lacking mainly due to the limitation of the method of increasing mitophagy, few examples suggest this strategy is worth a try. For example, overexpression Parkin led to increasing of mitochondrial activity and lifespan in Drosophila (69). Rye D et al. recently reported that treatment of urolithin A, a novel mitophagy induced, increased life span in C. elegans and improved age-related muscle function in mouse models (70).

\section{MOLECULAR MECHANISM OF MITOPHAGY}

\section{PINK1-parkin pathway}

While mitophagy mechanism has been systematically studied and the identified the important role of ATG genes in yeast (71), the regulatory pathway for mammalian mitophagy is still largely unknown. Studies on the molecular mechanisms of mitochondria in mammalian cells are mainly focused on the PINK1-Parkin pathway. According to studies conducted to date, in conditions where normal mitochondrial membrane potential $(\Delta \Psi \mathrm{m})$ is maintained, PINK1 protein is rapidly degraded, and Parkin is present in the protoplasm. However, when mitochondrial damage occurs, membrane potential decreases, PINK1 is stabilized by autophosphorylation, recruit of Parkin to mitochondria and increased ubiquitin E3 ligase enzyme activity leads to the induction of mitophagy (13). It has been identified that Parkin-mediated ubiquitination promotes intervention of additional factors, and direct phosphorylation of ubiquitin by PINK promotes intervention of Parkin and mitochondrial receptors.

Recent findings from intensive studies show that mitophagy is regulated by a complex signaling network involving various regulators, rather than a simple linear regulatory pathway. For example, recently Richard Youle's group published an interesting study investigating the function of several autophagy receptors in mitophagy of Hela cells (72). Through inactivating five autophagy receptors, TAX1BP1, NDP52, NBR1, p62, and OPTN (optineurin), in various combination by using gene editing techniques, they found only NDR52 and OPTN are actually involved in mitophagy. Interestingly, PINK1-mediated ubiquitin phosphorylation induces NDR52 and OPTN intervention but does not require the function of Parkin in this process. However, Parkin acts as an 'amplifier' to increase mitochondrial activity. This study well pointed out both the unique aspect of mitophagy mechanism and the requirement of further study that will clarify the exact functions of PINK1 and Parkin, which are important to understanding the precise molecular mechanism of mitophagy.

\section{PINK1-Parkin independent pathway}

On the other hand, there have been reports regarding PINK1 and Parkin-independent occurrence of mitophagy. In 2013, the Kagan Group reported that during rotenone-mediated mitophagy, cardiolipin lipid components present in the inner mitochondrial membrane are essential for transport to the outer membrane and that this occurs irrespective of PINK1 function (73). In the same year, the Ganley group found that mitophagy that is induced by treatment of deferiprone, an iron chelator, also occurred independently of the PINK1-Parkin pathway (74). Recently, the Gustafsson group published a 
remarkable study using PINK1-deficient mice (75). Upon application of myocardial infarction or mitochondrial uncoupler to heart tissue and myocardial cells of PINK1-deficient mice, the transfer of Parkin to mitochondria, the ubiquitination of mitochondrial proteins and the mitochondria was found to occur normally. These results suggest that in addition to the PINK1-Parkin pathway, additional Parkin migration and mitophagy activation pathways exist as a compensatory mechanism in case of PINK deficiency.

\section{Regulators for mitophagy induction}

To understand the physiological function of mitophagy and its role in disease development, it is an essential task to identify the regulatory pathway of mitophagy. Recent intensive studies have led to the identification of new regulatory genes and regulatory mechanisms. Mitochondrial receptors have also been found to contain several proteins, including NIX, BNIXP3, p62, and NBR1. More studies are required to investigate mitophagy-induced signals in physiological environments. In addition to hypoxia conditions, iron chelation has been reported to induce mitophagy. However, the molecular mechanism is yet unclear (74). Interestingly, there have been studies involving lipids as a mitophagy regulator. It has been reported that ceramide, a typical sphingolipid inducing apoptosis and senescence, induces mitophagy (76). In addition, cardiolipin, a phospholipid present in the inner mitochondrial membrane, migrates to the mitochondrial outer membrane to promote mitophagy and remove damaged mitochondria $(73,77)$. Intriguingly, while peroxidized cardiolipin induces apoptosis, non-peroxidized cardiolipin induces mitophagy to protect cells from apoptotic cell death (77).

Considerable attention has also been paid to the role of mitochondrial dynamics in mitophagy. Since an increase in mitochondrial fragmentation is observed in mitophagyinduced conditions, mitochondrial fission is thought to be a pre-requisite of mitophagy for mammalian cells. Consistent with this notion, it has been shown that Dynamin-related protein 1 (Drp1), which is a critical fission regulator, is essential for mitophagy in yeast (78). Drp1-deficient MEFs also exhibited a significant reduction of Parkin-mediated mitophagy (79). However, whether the increase in mitochondrial fission is sufficient for the induction of mitophagy remains unclear. In fact, Drp1-indendent mitophagy has been recently also reported (80). Gomes LC et al. showed previously that overexpression of a Fis1 mutant that induces mitochondrial fragmentation without mitochondria dysfunction did not induce mitophagy (81). Thus, in order to induce mitophagy, mitochondria fission seems to require an additional signal that may derive from mitochondrial dysfunction.

\section{CONCLUDING REMARKS}

Since Lemestars coined the term "mitophagy" in 2005, focused studies on mitophagy attained significant results including the discovery of the PINK1-Parkin pathway. Recent results of mitophagy analysis at the stage of development and cell differentiation suggest that mitophagy not only has a passive role in removing damaged mitochondria but also has the ability to alter the intracellular mitochondrial activity and further cell function actively. However, in order to understand the function of mitophagy, many questions remain to be answered. For example, what is the precise mechanism of PINK1-mediated mitophagy induction? What signaling pathways regulate mitophagy in addition to the PINK1-Parkin pathway? How are damaged mitochondria selectively removed? Why are human orthologues of yeast mitophagy-related genes unidentified? Can mitophagy dysfunction contribute to a variety of human diseases other than Parkinson's disease? Is the mitophagy signaling pathway consistent among many cell types and tissues? How are mitophagy, mitochondrial biosynthesis, and fusion-fission mechanisms connected? Are there substances that selectively increase mitophagy? Can activation of mitophagy improve mitochondrial function in vivo? Does activation of mitophagy benefit aging? Can activation of mitophagy be used to treat human diseases including degenerative brain diseases?

Fortunately, recent brilliant technological advances have given a positive outlook on mitophagy research. A prime example is the development of animal models and techniques for directly measuring mitophagy activity in living tissues (68). Identification of the molecular mechanism of mitophagy followed by its utilization to develop strategies for disease treatment is anticipated for the near future.

\section{ACKNOWLEDGEMENTS}

I apologize to the authors whose work could not be referenced due to space limitation. This research was supported by a grant from National Research Foundation of Korea (2016R1A2B200 8887) and Medical Research Center grant (2016R1A5A2007009).

\section{CONFLICTS OF INTEREST}

The authors have no conflicting financial interests.

\section{REFERENCES}

1. Taylor RW and Turnbull DM (2005) Mitochondrial DNA mutations in human disease. Nat Rev Genet 6, 389-402

2. Kang D and Hamasaki N (2005) Alterations of mitochondrial DNA in common diseases and disease states: aging, neurodegeneration, heart failure, diabetes, and cancer. Curr Med Chem 12, 429-441

3. Sun N, Youle RJ and Finkel T (2016) The Mitochondrial Basis of Aging. Mol Cell 61, 654-666

4. Baker MJ, Palmer CS and Stojanovski D (2014) Mitochondrial protein quality control in health and disease. $\mathrm{Br}$ J Pharmacol 171, 1870-1889

5. Burte F, Carelli V, Chinnery PF and Yu-Wai-Man P (2015) 
Disturbed mitochondrial dynamics and neurodegenerative disorders. Nat Rev Neurol 11, 11-24

6. Ashrafi G and Schwarz TL (2013) The pathways of mitophagy for quality control and clearance of mitochondria. Cell Death Differ 20, 31-42

7. Clark IE, Dodson MW, Jiang $C$ et al (2006) Drosophila pink1 is required for mitochondrial function and interacts genetically with parkin. Nature 441, 1162-1166

8. Park J, Lee SB, Lee S et al (2006) Mitochondrial dysfunction in Drosophila PINK1 mutants is complemented by parkin. Nature 441, 1157-1161

9. Narendra D, Tanaka A, Suen DF and Youle RJ (2008) Parkin is recruited selectively to impaired mitochondria and promotes their autophagy. J Cell Biol 183, 795-803

10. Zhu J, Wang KZ and Chu CT (2013) After the banquet: mitochondrial biogenesis, mitophagy, and cell survival. Autophagy 9, 1663-1676

11. Lemasters JJ (2014) Variants of mitochondrial autophagy: Types 1 and 2 mitophagy and micromitophagy (Type 3). Redox Biol 2, 749-754

12. Lu H, Li G, Liu L, Feng L, Wang $X$ and Jin H (2013) Regulation and function of mitophagy in development and cancer. Autophagy 9, 1720-1736

13. Eiyama A and Okamoto K (2015) PINK1/Parkin-mediated mitophagy in mammalian cells. Curr Opin Cell Biol 33, 95-101

14. Palikaras K, Lionaki E and Tavernarakis N (2015) Coordination of mitophagy and mitochondrial biogenesis during ageing in C. elegans. Nature 521, 525-528

15. Shin JH, Ko HS, Kang H et al (2011) PARIS (ZNF746) repression of PGC-1alpha contributes to neurodegeneration in Parkinson's disease. Cell 144, 689-702

16. Sandoval H, Thiagarajan P, Dasgupta SK et al (2008) Essential role for Nix in autophagic maturation of erythroid cells. Nature 454, 232-235

17. Al Rawi S, Louvet-Vallee S, Djeddi A et al (2011) Postfertilization autophagy of sperm organelles prevents paternal mitochondrial DNA transmission. Science 334, 1144-1147

18. Sato M and Sato K (2011) Degradation of paternal mitochondria by fertilization-triggered autophagy in C. elegans embryos. Science 334, 1141-1144

19. Wilson-Fritch L, Burkart A, Bell G et al (2003) Mitochondrial biogenesis and remodeling during adipogenesis and in response to the insulin sensitizer rosiglitazone. Mol Cell Biol 23, 1085-1094

20. Kita $T$, Nishida $H$, Shibata $H$, Niimi $S$, Higuti $T$ and Arakaki N (2009) Possible role of mitochondrial remodelling on cellular triacylglycerol accumulation. J Biochem 146, 787-796

21. Sin J, Andres AM, Taylor DJ et al (2016) Mitophagy is required for mitochondrial biogenesis and myogenic differentiation of $\mathrm{C} 2 \mathrm{C} 12$ myoblasts. Autophagy 12, 369-380

22. Takahashi $\mathrm{K}$ and Yamanaka S (2006) Induction of pluripotent stem cells from mouse embryonic and adult fibroblast cultures by defined factors. Cell 126, 663-676

23. Folmes CD, Nelson TJ, Martinez-Fernandez A et al (2011) Somatic oxidative bioenergetics transitions into pluripotency- dependent glycolysis to facilitate nuclear reprogramming. Cell Metab 14, 264-271

24. Prigione A, Fauler B, Lurz R, Lehrach $\mathrm{H}$ and Adjaye J (2010) The senescence-related mitochondrial/oxidative stress pathway is repressed in human induced pluripotent stem cells. Stem Cells 28, 721-733

25. Varum S, Rodrigues AS, Moura MB et al (2011) Energy metabolism in human pluripotent stem cells and their differentiated counterparts. PLoS One 6, e20914

26. Zeuschner D, Mildner $K$, Zaehres $H$ and Scholer HR (2010) Induced pluripotent stem cells at nanoscale. Stem Cells Dev 19, 615-620

27. Kubli DA and Gustafsson AB (2012) Mitochondria and mitophagy: the yin and yang of cell death control. Circ Res 111, 1208-1221

28. Djavaheri-Mergny M, Maiuri MC and Kroemer G (2010) Cross talk between apoptosis and autophagy by caspasemediated cleavage of Beclin 1. Oncogene 29, 1717-1719

29. Pagliarini V, Wirawan E, Romagnoli A et al (2012) Proteolysis of Ambra1 during apoptosis has a role in the inhibition of the autophagic pro-survival response. Cell Death Differ 19, 1495-1504

30. Kim MJ, Yoon JH and Ryu JH (2016) Mitophagy: a balance regulator of NLRP3 inflammasome activation. BMB Rep 49, 529-535

31. Kang R, Zeng L, Xie Y et al (2016) A novel PINK1- and PARK2-dependent protective neuroimmune pathway in lethal sepsis. Autophagy 12, 2374-2385

32. Kim MJ, Bae SH, Ryu JC et al (2016) SESN2/sestrin2 suppresses sepsis by inducing mitophagy and inhibiting NLRP3 activation in macrophages. Autophagy 12, 1272-1291

33. Cha MY, Kim DK and Mook-Jung I (2015) The role of mitochondrial DNA mutation on neurodegenerative diseases. Exp Mol Med 47, e150

34. Nah J, Yuan J and Jung YK (2015) Autophagy in neurodegenerative diseases: from mechanism to therapeutic approach. Mol Cells 38, 381-389

35. Valente EM, Abou-Sleiman PM, Caputo V et al (2004) Hereditary early-onset Parkinson's disease caused by mutations in PINK1. Science 304, 1158-1160

36. Kitada T, Asakawa S, Hattori N et al (1998) Mutations in the parkin gene cause autosomal recessive juvenile parkinsonism. Nature 392, 605-608

37. Greene JC, Whitworth AJ, Kuo I, Andrews LA, Feany MB and Pallanck LJ (2003) Mitochondrial pathology and apoptotic muscle degeneration in Drosophila parkin mutants. Proc Natl Acad Sci U S A 100, 4078-4083

38. Hsieh CH, Shaltouki A, Gonzalez AE et al (2016) Functional Impairment in Miro Degradation and Mitophagy Is a Shared Feature in Familial and Sporadic Parkinson's Disease. Cell Stem Cell 19, 709-724

39. Ashrafi G, Schlehe JS, LaVoie MJ and Schwarz TL (2014) Mitophagy of damaged mitochondria occurs locally in distal neuronal axons and requires PINK1 and Parkin. J Cell Biol 206, 655-670

40. Wang X, Winter D, Ashrafi G et al (2011) PINK1 and Parkin target Miro for phosphorylation and degradation to arrest mitochondrial motility. Cell 147, 893-906

41. Court FA and Coleman MP (2012) Mitochondria as a central sensor for axonal degenerative stimuli. Trends 
Neurosci 35, 364-372

42. Flatters SJ and Bennett GJ (2006) Studies of peripheral sensory nerves in paclitaxel-induced painful peripheral neuropathy: evidence for mitochondrial dysfunction. Pain 122, 245-257

43. Pareyson D, Piscosquito G, Moroni I, Salsano E and Zeviani M (2013) Peripheral neuropathy in mitochondrial disorders. Lancet Neurol 12, 1011-1024

44. Rizzo F, Ronchi D, Salani S et al (2016) Selective mitochondrial depletion, apoptosis resistance, and increased mitophagy in human Charcot-Marie-Tooth 2A motor neurons. Hum Mol Genet 25, 4266-4281

45. Chourasia AH, Boland ML and Macleod KF (2015) Mitophagy and cancer. Cancer Metab 3, 4

46. Cesari R, Martin ES, Calin GA et al (2003) Parkin, a gene implicated in autosomal recessive juvenile parkinsonism, is a candidate tumor suppressor gene on chromosome 6q25-q27. Proc Natl Acad Sci U S A 100, 5956-5961

47. Veeriah S, Taylor BS, Meng S et al (2010) Somatic mutations of the Parkinson's disease-associated gene PARK2 in glioblastoma and other human malignancies. Nat Genet 42, 77-82

48. Fujiwara M, Marusawa $\mathrm{H}$, Wang HQ et al (2008) Parkin as a tumor suppressor gene for hepatocellular carcinoma. Oncogene 27, 6002-6011

49. Letessier A, Garrido-Urbani S, Ginestier C et al (2007) Correlated break at PARK2/FRA6E and loss of AF-6/Afadin protein expression are associated with poor outcome in breast cancer. Oncogene 26, 298-307

50. Poulogiannis G, Mclntyre RE, Dimitriadi M et al (2010) PARK2 deletions occur frequently in sporadic colorectal cancer and accelerate adenoma development in Apc mutant mice. Proc Natl Acad Sci U S A 107, 15145-15150

51. Zhang C, Lin M, Wu R et al (2011) Parkin, a p53 target gene, mediates the role of p53 in glucose metabolism and the Warburg effect. Proc Natl Acad Sci U S A 108, 16259-16264

52. de Reynies A, Assie G, Rickman DS et al (2009) Gene expression profiling reveals a new classification of adrenocortical tumors and identifies molecular predictors of malignancy and survival. J Clin Oncol 27, 1108-1115

53. Fragoso MC, Almeida MQ, Mazzuco TL et al (2012) Combined expression of BUB1B, DLGAP5, and PINK1 as predictors of poor outcome in adrenocortical tumors: validation in a Brazilian cohort of adult and pediatric patients. Eur J Endocrinol 166, 61-67

54. Calvisi DF, Ladu S, Gorden A et al (2007) Mechanistic and prognostic significance of aberrant methylation in the molecular pathogenesis of human hepatocellular carcinoma. J Clin Invest 117, 2713-2722

55. Chourasia AH, Tracy K, Frankenberger C et al (2015) Mitophagy defects arising from BNip3 loss promote mammary tumor progression to metastasis. EMBO Rep $16,1145-1163$

56. Sowter HM, Ratcliffe PJ, Watson P, Greenberg $\mathrm{AH}$ and Harris AL (2001) HIF-1-dependent regulation of hypoxic induction of the cell death factors BNIP3 and NIX in human tumors. Cancer Res 61, 6669-6673

57. Zhang H, Bosch-Marce M, Shimoda LA et al (2008)
Mitochondrial autophagy is an HIF-1-dependent adaptive metabolic response to hypoxia. J Biol Chem 283, 10892-10903

58. Billia F, Hauck L, Konecny F, Rao V, Shen J and Mak TW (2011) PTEN-inducible kinase 1 (PINK1)/Park6 is indispensable for normal heart function. Proc Natl Acad Sci U S A $108,9572-9577$

59. Dorn GW 2nd (2010) Mitochondrial pruning by Nix and BNip3: an essential function for cardiac-expressed death factors. J Cardiovasc Transl Res 3, 374-383

60. Nakai A, Yamaguchi O, Takeda T et al (2007) The role of autophagy in cardiomyocytes in the basal state and in response to hemodynamic stress. Nat Med 13, 619-624

61. Gong G, Song M, Csordas G, Kelly DP, Matkovich SJ, Dorn GW 2nd (2015) Parkin-mediated mitophagy directs perinatal cardiac metabolic maturation in mice. Science 350, aad2459

62. Kubli DA, Zhang $X$, Lee $Y$ et al (2013) Parkin protein deficiency exacerbates cardiac injury and reduces survival following myocardial infarction. J Biol Chem 288, 915-926

63. Siddall HK, Yellon DM, Ong SB et al (2013) Loss of PINK1 increases the heart's vulnerability to ischemia-reperfusion injury. PLoS One 8, e62400

64. Huang C, Andres AM, Ratliff EP, Hernandez G, Lee P and Gottlieb RA (2011) Preconditioning involves selective mitophagy mediated by Parkin and p62/SQSTM1. PLoS One 6, e20975

65. Takamura A, Komatsu M, Hara T et al (2011) Autophagy-deficient mice develop multiple liver tumors. Genes Dev 25, 795-800

66. Glick D, Zhang W, Beaton M et al (2012) BNip3 regulates mitochondrial function and lipid metabolism in the liver. Mol Cell Biol 32, 2570-2584

67. Williams JA and Ding WX (2015) A Mechanistic Review of Mitophagy and Its Role in Protection against Alcoholic Liver Disease. Biomolecules 5, 2619-2642

68. Sun N, Yun J, Liu J et al (2015) Measuring In Vivo Mitophagy. Mol Cell 60, 685-696

69. Rana A, Rera M and Walker DW (2013) Parkin overexpression during aging reduces proteotoxicity, alters mitochondrial dynamics, and extends lifespan. Proc Natl Acad Sci U S A 110, 8638-8643

70. Ryu D, Mouchiroud L, Andreux PA et al (2016) Urolithin $A$ induces mitophagy and prolongs lifespan in C. elegans and increases muscle function in rodents. Nat Med 22, 879-888

71. Kanki T, Furukawa K and Yamashita S (2015) Mitophagy in yeast: Molecular mechanisms and physiological role. Biochim Biophys Acta 1853, 2756-2765

72. Lazarou M, Sliter DA, Kane LA et al (2015) The ubiquitin kinase PINK1 recruits autophagy receptors to induce mitophagy. Nature 524, 309-314

73. Chu CT, Ji J, Dagda RK et al (2013) Cardiolipin externalization to the outer mitochondrial membrane acts as an elimination signal for mitophagy in neuronal cells. Nat Cell Biol 15, 1197-1205

74. Allen GF, Toth R, James J and Ganley IG (2013) Loss of iron triggers PINK1/Parkin-independent mitophagy. EMBO Rep 14, 1127-1135 
75. Kubli DA, Cortez MQ, Moyzis AG, Najor RH, Lee $Y$ and Gustafsson AB (2015) PINK1 Is Dispensable for Mitochondrial Recruitment of Parkin and Activation of Mitophagy in Cardiac Myocytes. PLoS One 10, e0130707

76. Sentelle RD, Senkal CE, Jiang W et al (2012) Ceramide targets autophagosomes to mitochondria and induces lethal mitophagy. Nat Chem Biol 8, 831-838

77. Ren M, Phoon CK and Schlame M (2014) Metabolism and function of mitochondrial cardiolipin. Prog Lipid Res 55, $1-16$

78. Kanki T, Wang K and Klionsky DJ (2010) A genomic screen for yeast mutants defective in mitophagy. Auto- phagy 6, 278-280

79. Tanaka A, Cleland MM, Xu S et al (2010) Proteasome and p97 mediate mitophagy and degradation of mitofusins induced by Parkin. J Cell Biol 191, 1367-1380

80. Yamashita SI, Jin X, Furukawa K et al (2016) Mitochondrial division occurs concurrently with autophagosome formation but independently of Drp1 during mitophagy. J Cell Biol 215, 649-665

81. Gomes LC and Scorrano L (2008) High levels of Fis1, a pro-fission mitochondrial protein, trigger autophagy. Biochim Biophys Acta 1777, 860-866 